\title{
Aufbereitung von chirurgischen Instrumenten und Implantaten
}

\author{
Nikolaus Schwarz, Manfred Windisch, Helga Ruppnig
}

\begin{abstract}
Zusammenfassung
Wundinfektionen nach Operation gehören zu den grundsätzlich, aber nicht zu $100 \%$ vermeidbaren Patientenrisiken. Ein diffiziles organisatorisches und maschinelles System ist im Krankenhaus erforderlich, um der Entstehung von Infektionen vorzubeugen. Exakte Arbeitsvorschriften regeln die einzelnen Prozesse. Kein System kann aber Arbeitsdisziplin und Genauigkeit in der Tätigkeit aller Beteiligten ersetzen. Die Aufbereitung von Instrumenten und Implantaten gliedert sich
\end{abstract}

in drei Schritte: Reinigung und Desinfektion, Pflege und Prüfung, Verpackung und Sterilisation. Jeder Schritt wird dokumentiert, so dass der Weg eines Instrumentes zum Patienten und die einzelnen Aktionen nachvollziehbar sind. Jeder automatische Arbeitsprozess wird laufend auf Effektivität überprüft und ebenfalls dokumentiert. Das Ziel ist die weitestmögliche Sicherheit vor Infektionsübertragung und postoperativer Wundinfektion für Patienten und Krankenhauspersonal.

\section{Einleitung}

Der Operationssaal muss mit sterilen chirurgischen Instrumenten versorgt werden und es müssen auch die in der Unfallchirurgie/Orthopädischen Chirurgie häufig benötigten Implantate für Osteosynthesen steril bereitgestellt werden. Infektionen, die im Krankenhaus erworben werden - darunter fallen natürlich auch alle postoperativen Wundinfektionen - bilden nach wie vor ein enormes medizinisches und finanzielles Problem. Die Zahl postoperativer Wundinfektionen in Deutschland wird pro Jahr auf 128000 geschätzt. Eine BSE-Übertragung (Creutzfeld-Jakob-Krankheit) durch mangelhaft sterilisierte Instrumente wurde aus England und aus Australien berichtet.

Die Aufbereitung von Instrumenten und Implantatmaterialien erfolgt in validierten, überprüfbaren und auch ständig überprüften Abläufen. Die Gestaltung einer geordneten Aufbereitung ist notwendig, um benützte chirurgische Instru-

OP-JOURNAL 2005; 21: 4-9

(c) Georg Thieme Verlag KG Stuttgart · New York mente ohne Gefährdung von Patienten oder Personal aus dem OP-Bereich zu transportieren, zu reinigen, zu sterilisieren und wieder zur Verfügung zu stellen. Jeder Verfahrensschritt erfolgt so weit als technisch möglich automatisiert, um einen möglichst hohen Schutz vor Fehlern zu haben. Jede Phase des Prozesses kann sofort, aber auch rückwirkend auf ihre Effektivität geprüft werden. Das ist etwa bei Auftreten von Infektionsserien von direkter praktischer Bedeutung. Gleichzeitig werden die Instrumente bei diesem Zyklus auf Schäden bzw Funktion geprüft, sie werden gewartet und gepflegt, nicht reparierbare Teile werden ausgewechselt. Die Lagerung der Instrumente und Implantate bis zum Gebrauch ist so zu gestalten, dass eine Schädigung, welche die Sterilität gefährdet, mit Sicherheit vermieden wird. Der rasche $\mathrm{Zu}-$ griff auf alle Instrumente ist ein wichtiger, praktischer Gesichtspunkt der Lagerhaltung.

Aubereitung von Instrumenten: Reinigung, Desinfektion, Spülung, Trocknung - Prüfung auf Sauberkeit und Unversehrtheit, Pflege und Instandsetzung, Funktionsprüfung, Verpackung, Kennzeichnung, Sterilisation

\section{Begriffsbestimmungen}

Reinigung

Unter Reinigung versteht man die Entfernung von Verunreinigung unter der Verwendung von Wasser, dem ein Reinigungsmittel (Detergens) beigefügt ist. Ein hoher Grad an Sauberkeit ist Vorbedingung für eine effektive Desinfektion und Sterilisation. Eine Abtötung von Keimen oder anderen Mikroorganismen ist nicht beabsichtigt.

\section{Desinfektion}

Desinfektion ist die gezielte Verminderung von Mikroorganismen durch physikalische oder chemische Maßnahmen. Es ist nicht das Ziel der Desinfektion, alle Keime abzutöten, sondern es soll erreicht werden, dass durch eine Reduktion der Keimzahl die Infektkette unterbrochen wird. Die Wirkung eines Desinfektionsmittels ist abhängig von seiner Zusammensetzung, seiner Konzentration und der Einwirkzeit. Es gibt thermische (z.B. Heißwasser) und chemische Desinfektionsverfahren. Täglich geübtes Beispiel in Krankenhaus oder Arztordination ist die Händedesinfektion.

Es werden folgende mikrobiologischen Wirkungsbereiche von Desinfektionsmitteln unterschieden:

A - Abtötung von Bakterien, Pilzen und deren Sporen,

B - Inaktivierung von Viren,

C - Abtötung von bakteriellen Sporen einschließlich Milzbrand,

D - Abtötung von bakteriellen Sporen einschließlich Gasbrand und Wundstarrkrampf

Die Wirkungsbereiche A und B werden durch Desinfektionsmaßnahmen abgedeckt. Hingegen sind zur Abtötung von Sporen Sterilisationsmaßnahmen durchzuführen. Desinfektionsgüter sind Materialien, die nach Reinigung und Desinfek- 


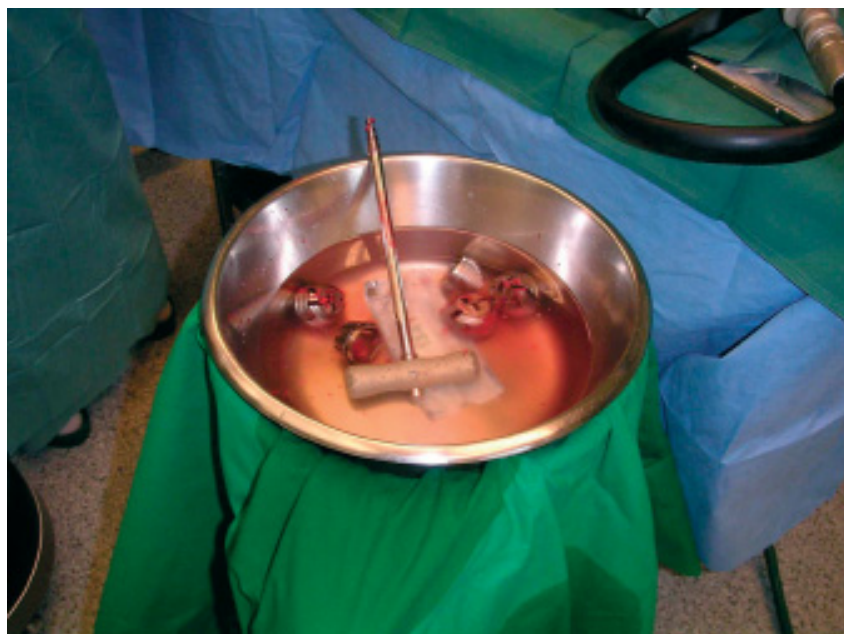

Abb.1 Behälter mit steriler Flüssigkeit zur Grobreinigung verschmutzter Instrumente während und zum Abschluss der Operation.

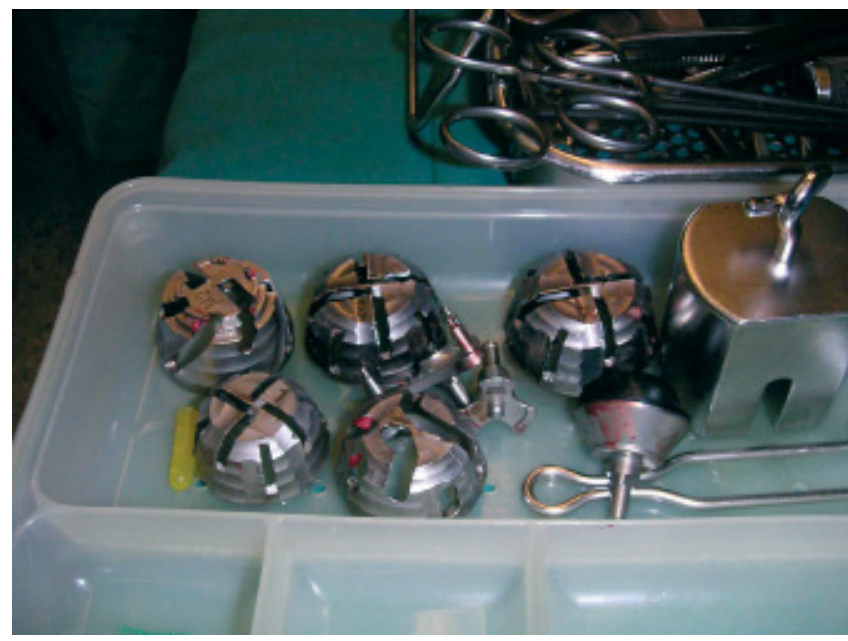

Abb. 2 Derart gereinigte Instrumente am Instrumentiertisch vor dem Abtransport in die Zentralsterilisation. tion einsetzbar sind. Dazu gehört etwa Anästhesiezubehör, d.s. semikritische Medizinprodukte.

Desinfektion ist die gezielte Verminderung von Mikroorganismen durch physikalische oder chemische Maßnahmen.

\section{Sterilisation}

Sterilisation ist das Abtöten aller vorhandenen Mikroorganismen. Absolute Keimfreiheit wird für alle Gegenstände gefordert, die mit sterilen Objekten, mit der Blutbahn oder mit Organen in Kontakt kommen. Im Unterschied zur Desinfektion richtet sich die Sterilisation nicht gegen bestimmte Krankheitserreger, sondern sie richtet sich gegen alle vorhandenen Mikroorganismen mit ihren Dauerformen (Sporen). Es gibt physikalische und chemische Sterilisationsverfahren. Sterilgüter sind Materialien, die nur absolut keimfrei zum Einsatz kommen dürfen. Sie werden den kritischen Medizinprodukten zugeordnet.

Sterilisation ist das Abtöten aller vorhandenen Mikroorganismen.

\section{Bautechnische Anforderungen}

Die Instrumentenaufbereitung soll zentral erfolgen. Der Arbeitsablauf sieht grundsätzlich vor, dass eine strikte räumliche und organisatorische Trennung einen direkten oder indirekten Kontakt zwischen unsterilem und sterilem Gut vermeidet und er sieht einen Einbahnablauf vom unsterilen zum sterilen Bereich vor. Die Funktionseinheiten müssen räumlich getrennt sein und sollen nur über Schleusensysteme erreichbar sein. Desinfektionsautomaten sind in diesem Konzept vorzugsweise als Durchladegeräte vorgesehen, die in Trennwände zwischen reinem und unreinem Bereich eingebaut werden und die von der unreinen Seite beladen und von der reinen Seite entladen werden können.

\section{Instrumentenkreislauf - Schritte der Aufbereitung}

\section{Reinigung und Desinfektion}

Die fachgerechte Aufbereitung der Instrumente beginnt bereits im OP vor oder bei deren Ablage. Diese Tätigkeit wird in den meisten Fällen von der instrumentierenden Schwester/Beidienst übernommen, welche OP-Mantel und Handschuhe dazu nicht ablegen. Starke Verschmutzungen von Instrumenten sind noch am Operationstisch zu beseitigen (Abb.1 und 2). Nach dieser Vorreinigung werden die Instrumente trocken in Siebschalen abgelegt. Diese sog. Trockenentsorgung ist die Methode der Wahl. Feuchtigkeit schadet den Instrumenten durch Korrosion und bietet potenziellen Erregern ein geeignetes Milieu. Lediglich in ausgewählten Fällen ist auch eine Nassentsorgung angezeigt. Hierbei werden die Instrumente aus Gründen des Personalschutzes in Reinigungs- bzw. Desinfektionslösungen eingelegt, wenn nachfolgend eine manuelle Aufbereitung erforderlich ist. Die Zuführung kontaminierter Instrumente zur Reinigung hat so rasch als möglich zu erfolgen

Es ist zu vermeiden, dass die Siebschalen übervoll sind oder dass Instrumente übereinander gelagert sind (Abb.3). Passiert das, kommt es zur Ausbildung sogenannter Spülschatten und damit zur unvollständigen Reinigung. Instrumente mit Gelenken werden geöffnet, zerlegbare Instrumente werden in Einzelteilen eingebracht. Die Siebschalen werden in verschließbaren Behältern transportiert, um eine Kontamination der Umgebung während des Transportes zu vermeiden. Die Siebschalen sollen auch direkt in die Waschmaschinen passen.

Die Desinfektionsverfahren müssen den Wirkungsbereich A und B haben. Den chemothermischen Verfahren ist wegen der zuverlässigeren Wirksamkeit der Vorrang vor chemischen Desinfektionsverfahren zu geben. Aldehyde etwa führen zur Fixierung von Proteinen, welche die Sterilisationsleistung beeinträchtigt.

Die Desinfektion/Dekontamination kann mithilfe physikalischer oder chemischer Verfahren durchgeführt werden. Grundsätzlich sind aus Sicherheitsgründen physikalische Maßnahmen zu bevorzugen. Physikalische Desinfektionsverfahren sind beispielsweise Pasteurisieren (Temperatur $62-150^{\circ} \mathrm{C}$, Wirkungsbereich A) oder Dampf $\left(75-105^{\circ} \mathrm{C}\right.$, Wirkungsbereich $A B C$ ). Chemische Desinfektionsmittel werden eingesetzt, wenn die Objekte hitzeempfindlich sind (Abb.4). Solche thermolabilen Produkte sind etwa optische Systeme oder Messsonden.

Die Wirksamkeit von Desinfektionsmitteln, die im Krankenhaus eingesetzt werden, muss nachgewiesen sein. Es dürfen nur Mittel verwendet werden, die in der DGHM-Liste (Deutsche Gesellschaft 


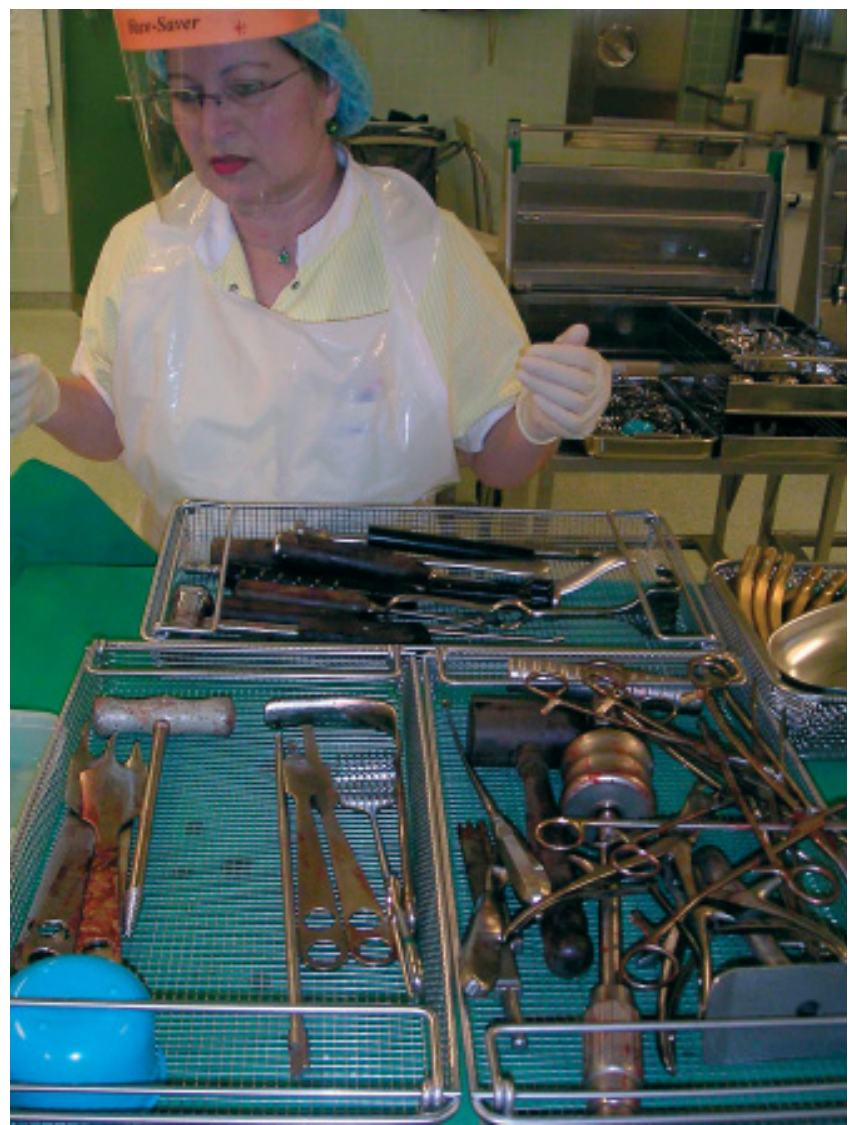

für Hygiene und Mikrobiologie) erscheinen.

Reinigung und Desinfektion chirurgischer Instrumente erfolgen vorzugsweise maschinell, weil dies die Verletzungsgefahr vermindert, die Kontamination der Umgebung reduziert und weil der Ablauf standardisiert ist. Ausnahmsweise kommt bei Instrumenten oder Geräten, die kompliziert gebaut sind, um in Waschmaschinen eingelegt zu werden, wie etwa Bohrmaschinen, eine manuelle Reinigung zur Anwendung. Bei diesen Tätigkeiten ist eine Schutzkleidung zu tragen (Maske mit Augenschutz, Handschuhe, Plastikschürze) (Abb.3).

Die Bildung von Reaktionsprodukten und Rückständen verwendeter Reinigungsund Desinfektionsmittel muss ausgeschlossen sein. Die abschließende Spülung erfordert entmineralisiertes Wasser, um Kristallbildungen auf den Instrumenten $z u$ vermeiden.

Besonders empfindliche Instrumente, wie sie beispielsweise in der minimalinvasiven Chirurgie Verwendung finden, müssen sofort dekontaminiert werden, weil sie durch eingetrocknete Flüssigkeit verkleben könnten. Sie werden vor Ort
Abb. 3 Sortieren der grob gereinigten Instrumente und Lagerung in Siebschalen. Bei dieser Tätigkeit ist Schutzkleidung zu tragen.

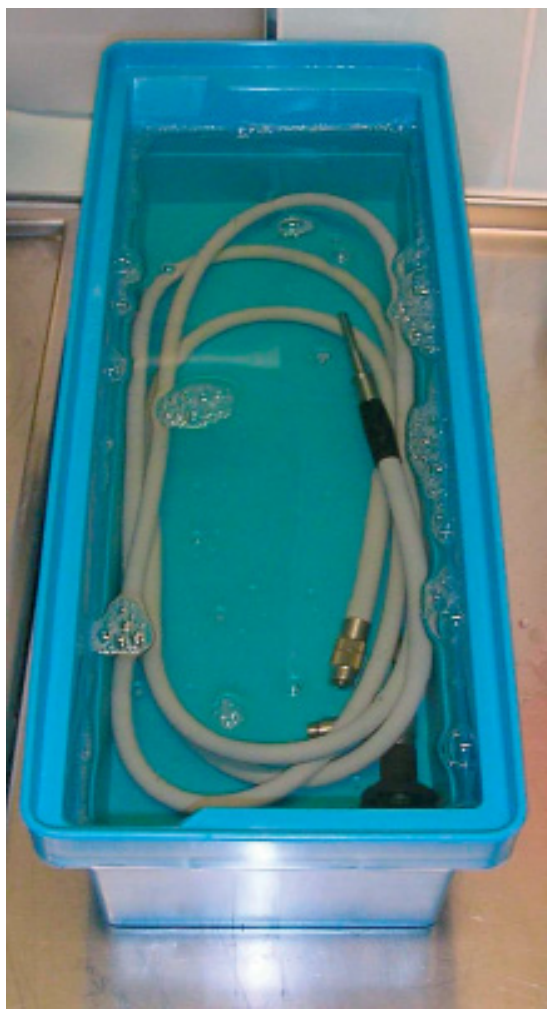

Abb. 4 Chemische Desinfektion von Lichtkabeln und Optiken.

kann. Instrumente mit Roststellen und Verunreinigungen oder solche mit mechanischen Schäden werden aussortiert. Anhaftende Verschmutzungen können darunter fixierte Keime schützen, so dass diese die Sterilisation überleben können.

Die Zuführung kontaminierter Instrumente zur Reinigung hat so rasch als möglich zu erfolgen.

Reinigung und Desinfektion erfolgen bevorzugt in automatisch gesteuerten Maschinen mittels thermischer Verfahren.

\section{Instrumentenpflege}

Instrumente und Maschinen, die zur Sterilisation vorbereitet sind, werden einer Funktionskontrolle unterzogen: Gelenke müssen leichtgängig sein, Ratschen müssen einrasten, Verschlüsse müssen funktionieren, bei schließenden Instrumenten wie Pinzetten dürfen die beiden Branchen nicht aneinander vorbei gehen. Die Gelenkflächen werden auf Restverschmutzung und Abrieb untersucht, alle Lumina werden kontrolliert, Funktionsüberprüfung aller rotierenden oder motorgetriebenen Instrumente. Die Sperren der Instrumente sollen nur im ersten Zahn geschlossen werden, weil ein stärkerer Druck auf Dauer zu Risskorrosionen und unvollständiger Sterilisation führen
Intakte und saubere Instrumente werden mit medizinischem Öl behandelt. Dies betrifft vor allem alle Gelenke. Ebenso sind zu diesem Zeitpunkt Maschinen nach Vorschrift zu warten und gegebenenfalls zu ölen. Überschüssiges Öl ist mit fusselfreien Tüchern zu entfernen. Kunststoffoberflächen sollen nicht mit Öl behandelt werden.

Spitze oder scharfe Instrumente, die die Sterilgutverpackung beschädigen könnten, sind entsprechend zu lagern oder einzuwickeln.

Fabrikneue Instrumente sollen vor dem ersten Gebrauch mehrfach gereinigt und sterilisiert werden, um Verunreinigungen aus der Produktion mit Sicherheit zu entfernen.

Die Instrumente werden in Sieben oder Containern nach Inhaltslisten zusammengestellt, verpackt und der Sterilisation zugeführt. 
Instrumente und Maschinen, die zur Sterilisation vorbereitet werden, müssen gleichzeitig einer Funktionskontrolle unterzogen werden.

\section{Sterilgutverpackung}

Die Verpackung besteht aus mechanischer Schutzverpackung, Sterilverpackung und ggf. einer Lager/Transportverpackung.

Die Sterilverpackung dient in erster Linie dazu, den Sterilisationsprozess zu ermöglichen und den Inhalt vor Kontamination für die Dauer der Lagerung zu schützen. Sie muss darüber hinaus einen gefahrlosen Transport des Sterilgutes erlauben und sie muss so gestaltete sein, dass die Entnahme des Inhaltes im OP ohne Gefahr einer Kontamination vorgenommen werden kann.

Die Verpackung muss Informationen über Inhalt, Sterilisationsdatum, Ablaufdatum und die Chargennummer sowie einen Sterilisationsindikator enthalten.

Sterilgutcontainer sind Metallbehälter, in welchen Instrumentensets in Siebkörben sterilisiert und aufbewahrt werden. Ventilcontainer sind wartungsfrei, solange keine mechanische Schädigung vorliegt. Sie werden nach Gebrauch desinfiziert. Die Siebkörbe können extra in Tuch eingepackt werden. Die Container müssen in regelmäßigen Abständen gereinigt, gewartet und desinfiziert werden.

Bei Einmalmaterialien zur Verpackung handelt es sich um Papier, Textilien oder Kunststoffe oder eine Kombination aus diesen. Die Verpackung mit Sterilisationspapier erfolgt in zwei oder drei Schichten Textil-Papier-Papier. Häufig kommen Klarsichtverpackungen zur Anwendung, die aus Papier und aufgeschweißten Kunststofffolien bestehen.

Das Folienschweißgerät ist bei Inbetriebnahme des ausgeschalteten Gerätes, sonst alle 24 Stunden zu überprüfen. Es wird dazu eine Testfolie bearbeitet, die erreichte Temperatur kontrolliert $\left(180^{\circ} \mathrm{C}\right)$, die Foliennaht auf Fehler untersucht und die Testfolie mit Datum und Namen des Untersuchers versehen und abgelegt.

\section{Sterilisation}

Voraussetzung für eine sichere Sterilisation ist die sorgfältige Reinigung der Produkte. Im Wesentlichen stehen Dampf-,
Gas- und Strahlensterilisation als Verfahren zur Verfügung. Reiner gesättigter Wasserdampf ist das sicherste Verfahren und daher wird die Dampfsterilisation allen anderen Sterilisationsmethoden vorgezogen. Gearbeitet wird bei einer Sterilisationstemperatur von $121^{\circ} \mathrm{C}$ und einer Einwirkzeit von mindestens 15 Minuten oder bei $134^{\circ} \mathrm{C}$ und mindestens 3 Minuten. Ein durchschnittlicher Sterilisationsvorgang dauert rund 70 Minuten, eine komplette Wiederaufbereitung etwa 3 Stunden. Um diese Temperaturen zu erreichen, wird mit Reindampf - ohne Luftbeimengung - bei erhöhtem Druck gearbeitet. Es muss also als erster Verfahrensschritt Luft aus dem Sterilisator evakuiert werden.

Die Beladung des Sterilisators hat gewisse Kriterien zu erfüllen (Abb.5): Leichte und einzeln verpackte Güter sind oben einzuordnen; das Sterilisiergut darf die Kammerwandung nicht berühren; einzeln verpacktes Gut soll senkrecht eingebracht werden, verpackte Gefäße werden mit der Öffnung nach unten gelagert. Der Sterilisationsvorgang und der Inhalt einer Charge wird dokumentiert (Abb.6).

Nach Abschluss der Sterilisationsvorganges ist das Gut auf Sauberkeit, Trockenheit und Unversehrtheit zu prüfen, der Vorgang selbst ist anhand der Prozessdaten zu überprüfen. Bei Entnahme heißen Sterilgutes ist $\mathrm{zu}$ beachten, dass sich durch rasche Abkühlung etwa in Zugluft Kondenswasser bilden und dieses zur Befeuchtung der Verpackung führen kann. Sterilgut mit feuchter Verpackung ist als unsteril anzusehen und einer neuerlichen Sterilisation zuzuführen.
Sterilisiertes und nicht sterilisiertes Gut muss eindeutig unterscheidbar sein.

Die Aufbereitung der Instrumente und Implantate kann sich auf die erneute Verpackung und Sterilisation beschränken, wenn eine Kontamination und Beschädigung ausgeschlossen ist, wenn das Gut etwa nicht verwendet wurde, sondern nur ein Instrument aus einer Box entnommen werden musste.

Die Entnahme des Sterilgutes erfolgt ebenfalls nach gewissen Vorschriften: Schutzkleidung, Händedesinfektion; Kontrolle der Dokumentation; 10 Minuten abkühlen lassen; Kontrolle auf Feuchtigkeit oder Beschädigung jeder einzelnen Verpackung und gleichzeitig Kontrolle der Sterilisationsindikatoren; Chargenkontrolle.

Nicht hitzebeständige Materialien und Geräte werden durch Ethylenoxid (EO) oder Formaldehyd (FA) gassterilisiert. Beide Gase sind toxisch und sind als Gefahrenstoff eingestuft. Plasmasterilisation ist eine Alternative zu Gas, weil diese mit niedrigen Temperaturen von $37^{\circ} \mathrm{C}$ bis $45^{\circ} \mathrm{C}$ arbeitet.

Strahlensterilisation findet im Krankenhausbereich keine Anwendung, die sogenannte Blitzsterilisation ist nicht mehr zulässig.

Die Dampfsterilisation arbeitet mit einer Temperatur von $121^{\circ} \mathrm{C} />15$ Minuten oder $134^{\circ} \mathrm{C} />3$ Minuten.

Nach Abschluss des Sterilisationsvorganges ist das Gut auf Sauberkeit, Trockenheit und Unversehrtheit zu prüfen.

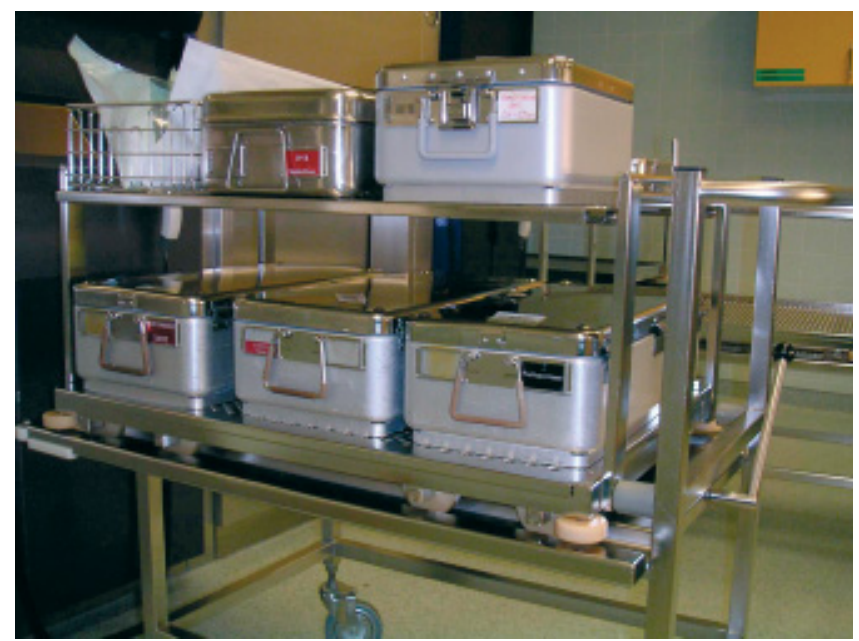

Abb. 5 Fertig beladener Sterilisationswagen. 


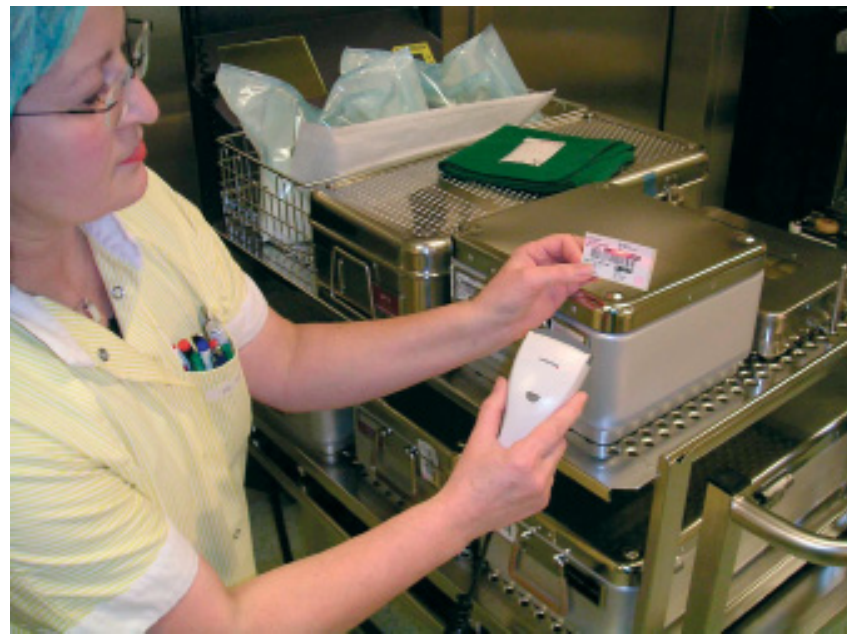

Abb. 6 Erfassen des Chargeninhalts mittels Barcode-Laser.

\section{Endoskopische Instrumente}

Neben Einmalinstrumenten sind in der Endoskopie zerlegbare und nicht zerlegbare Instrumente mit/ohne Spülanschluss $\mathrm{zu}$ unterscheiden. Instrumente, welche nicht zerlegbar sind und keine Möglichkeit zur Innenreinigung durch Spülung bieten, sind lt. Medizinproduktegesetz nicht wieder verwendbar.

Bei den anderen Geräten werden unmittelbar nach Gebrauch Hohlräume mit steriler Lösung durchgespült, Blut- und Gewebereste entfernt und die Instrumente zerlegt. In der Waschmaschine kann diese Reinigung ebenfalls erfolgen. Dazu werden die Geräte auf einen MIC-Aufsatz aufgesteckt (Abb. 7). Sie werden dann anschließend der Sterilisation zugeführt.

\section{Sterillagerung}

Sterile Güter sind in einem separaten Raum mit konstanter Temperatur (15- $25^{\circ} \mathrm{C}$ ) zu lagern (Sterilgutzone). Dieser darf nur in Schutzkleidung betreten werden. Die Sterilgüter müssen trocken gelagert werden, Feuchtigkeit führt zur Kontamination. Instrumente in durchnässten oder feuchten Verpackungen sind deshalb als unsteril zu betrachten. Maßnahmen, die zu Verletzungen von Verpackungen führen können, wie Anbringen von Gummiringen oder das Stapeln von Packungen, sind zu unterlassen. Die Lagerdauer richtet sich unter anderem nach der Verpackung und der Art des sterilen Materials. In offenen Regalen ist eine Lagerdauer von 12 Wochen für Container und Tuch-Papier-Papier-Verpackung oder Klarsichtverpackungen anzusetzen. Bei Lagerung im Schrank kann die Lagerdauer bei Zweifachverpackung 6 Monate betragen. Aus praktischen Gründen ist das Sterillager übersichtlich und leicht erreichbar zu organisieren.

Die Kontrolle der Ablaufdaten im Sterillager erfolgt wöchentlich.

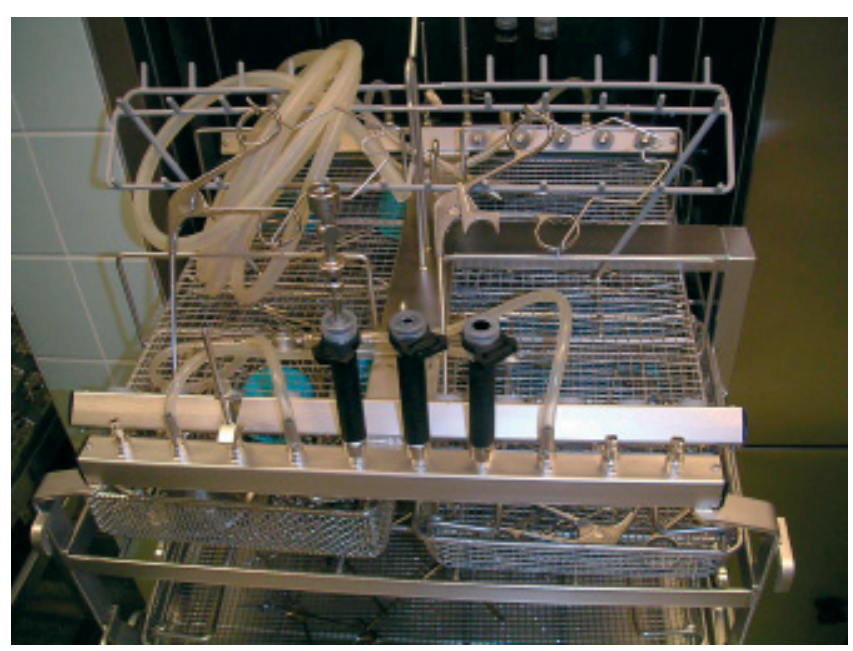

Abb. 7 Spülaufsätze für die Reinigung von Instrumenten für minimalinvasive Chirurgie.
Sterile Güter sind in einem separaten Raum, der nur in Schutzkleidung betreten werden darf, mit konstanter Temperatur $\left(15-25^{\circ} \mathrm{C}\right)$ zu lagern.

\section{Validierung, Kontrollen und Dokumentation}

Gemäß der Medizinprodukte-Betreiberverordnung sind Reinigung, Desinfektion und Sterilisation von Medizinprodukten mit geeigneten, validierten Verfahren so durchzuführen, dass der Erfolg dieser Verfahren nachvollziehbar gewährleistet und die Sicherheit von Patienten, Anwendern und Dritten nicht gefährdet ist. Mit der Validierung der Arbeitsprozesse werden auch die entsprechenden Parameter definiert, die eine Leistungsbeurteilung ermöglichen.

Die Reinigungskontrolle umfasst Sichtkontrollen auf Sauberkeit bei jeder Charge und Stichproben mittels Proteinnachweistests, gegebenenfalls auch eine Prüfung der Reinigungswirkung mittels Reinigungsindikatoren in regelmäßigen $\mathrm{Ab}$ ständen.

Die Desinfektionswirkung wird durch Kontrolle der Einhaltung der Desinfektionsparameter bei jeder Charge anhand des Ist-Soll-Vergleiches überprüft. $\mathrm{Zu}-$ sätzlich können geräteunabhängige Temperaturlogger zum Einsatz kommen.

Neben der ständigen Kontrolle auf Trübung gewährleistet die wöchentliche Prüfung der Leitfähigkeit und die $\mathrm{pH}-$ Messung eine entsprechende Wasserqualität.

Sterilisationsverfahren werden bei rückstandsfrei gereinigten Instrumenten validiert, ebenso maschinelle Reinigungsund Desinfektionsverfahren. Dies trifft nicht auf manuelle Reinigung und Desinfektion zu. Manuelle Verfahren sollten deshalb nur im Ausnahmefall angewandt werden und müssen nach dokumentierten Standardarbeitsanweisungen erfolgen.

Die Überprüfung von Sterilisatoren erfolgt durch einen Vakuumtest (dieser überprüft die Dichtheit der Kammer) und den Bowie-Dick-Test (dieser stellt die Dampfpenetration fest und soll am Beginn jedes Tages gemacht werden). Der Test arbeitet mit Farbindikator, der Testbogen wird an die ungünstigste Stelle, meist in die unterste Etage, gelagert. 


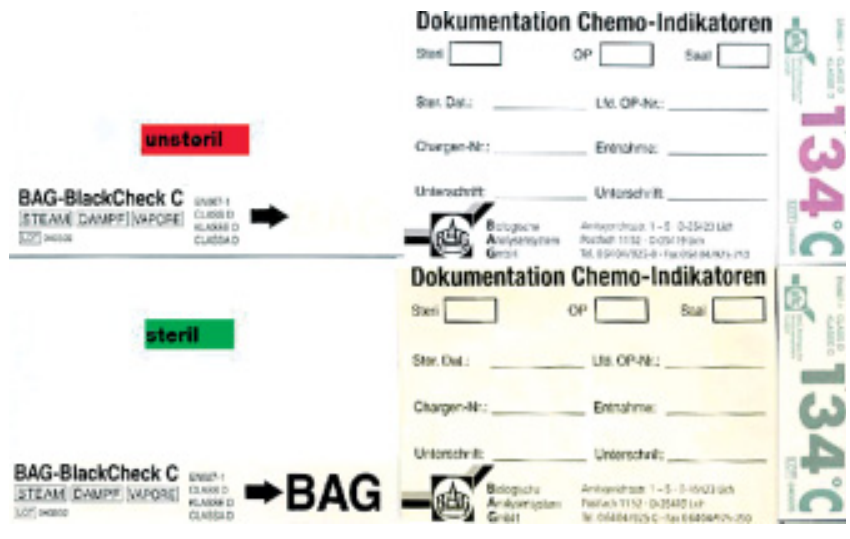

Abb.8 Farbindikatoren für Dampf-Sterilisatoren.

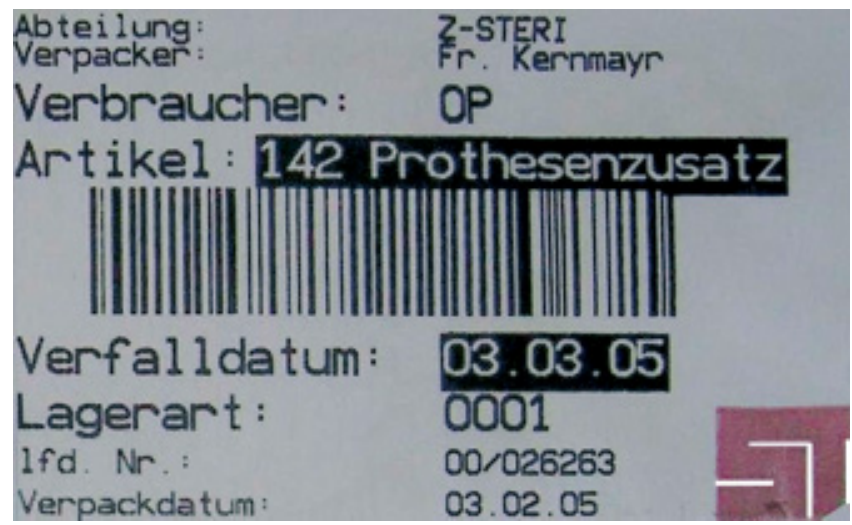

Abb.9 Auszug aus der Pflegedokumentation, wie sie im OP für jeden Patienten angelegt wird. Jedes Implantat und jede sterilisierte Kassette wird mittels einer Abziehfolie dokumentiert. Auf dieser sind die Artikelbezeichnung, das Verfalldatum, die Lagerart, die laufende Nummer, das Verpackdatum und der Verpacker registriert.
Für eine fachgerechte Dokumentation und Produktfreigabe des Sterilisationsgutes ist außerdem die Aufzeichnung der Druck- und Temperaturwerte sowie der Zeit erforderlich. Jedes Mal, wenn die Wirksamkeit des Verfahrens oder die Integrität der Verpackung infrage gestellt werden muss (nasses Sterilgut, aufgebrochene Packungen, unzureichender Farbumschlag chemischer Indikatoren), muss das behandelte Gut als unsteril betrachtet werden.

Die Freigabe des Gutes erfolgt, wenn die bei der Aufbereitung ermittelten Prozessparameter mit dem Validierungsprotokoll übereinstimmen.

Neben dem täglich durchgeführten Vakuumtest und Bowie-Dick-Test sollten weitere wöchentliche bzw. monatliche Tests und Wartungsarbeiten entsprechend den Vorgaben des Sterilisationsherstellers (z.B. Türdichtungen, sonstige Sicherheitstests) erfolgen.

Eine Revalidierung durch eine fachliche geeignete Stelle sollte jährlich stattfinden.
Für diese Routinekontrollen muss ein Plan erstellt werden, der eine Dokumentation der durchgeführten Tests und Kontrollen beinhaltet.

Zur Bestätigung eines einwandfreien Sterilisationszyklusses bzw. zur laufenden Kontrolle der Dampfqualität (nicht kondensierbare Gase, Änderungen der Dampfqualität im Laufe des Tages) wird jeder Charge ein chemisches Chargenkontrollsystems beigegeben (Abb.8). Diese chemischen Indikatoren erlauben durch Farbumschlag eine Unterscheidung ziwschen sterilisiertem und nicht sterilisiertem Gut. Durch ein geeignetes Dokumentationssystem ist sicherzustellen, dass jeweils pro Patient Details über den Aufbereitungsprozess der bei diesem Patienten verwendeten Materialien lückenlos rückverfolgt werden können (Abb.9). Die Dokumentation ist für die Dauer von 10 Jahre aufzubewahren.

Die Dokumentation muss beinhalten: Chargennummer (Hinweis auf Sterilisator, Sterilisierdatum), Aufzeichnung von Routineüberprüfungen wie Vakuumtest und Bowie-Dick-Test.

\section{Literatur}

1 Arbeitskreis „Krankenhaushygiene“ der AWMF: Hygiene in Klinik und Praxis. mhp Verlag Wiesbaden (2004)

2 http://www.innovations-report.de/html/ berichte/medizin

3 http://www.medicon.de/jsp/tree

${ }^{4}$ http://rki.de

${ }^{5}$ http://www.uni-essen.de/krankhaushygiene/skripte/desinfektion

6 NN: Anforderungen an die Hygiene bei Aufbereitung von Medizinprodukten. Bundesgesundheitsbl-Gesundheitsforsch-Gesundheitsschutz 44: 1115-1126 (2001)

7 ÖGSV, Leitlinie für die Prüfung, Validierung und Überwachung von maschinellen Reinigungs-/Desinfektionsverfahren für Medizinprodukte (2004) www.oegsv.com

Univ.-Prof. Dr. med. Nikolaus Schwarz Ärztlicher Leiter

Dr. med. Manfred Windisch

Sekundararzt

Helga Ruppnig

Leitende OP-Schwester

Unfallkrankenhaus

Waidmannsdorferstr. 35

A-9021 Klagenfurt 